\title{
RESEARCH
}

Open Access

\section{Mitochondrial dysfunction mediated through dynamin-related protein 1 (Drp1) propagates impairment in blood brain barrier in septic encephalopathy}

Bereketeab Haileselassie ${ }^{1,2^{*}+}$ D, Amit U. Joshi ${ }^{1^{*}+}$, Paras S. Minhas ${ }^{3}$, Riddhita Mukherjee ${ }^{1,2}$, Katrin I. Andreasson ${ }^{3}$ and Daria Mochly-Rosen ${ }^{1}$

\begin{abstract}
Background: Out of the myriad of complications associated with septic shock, septic-associated encephalopathy (SAE) carries a significant risk of morbidity and mortality. Blood-brain-barrier (BBB) impairment, which subsequently leads to increased vascular permeability, has been associated with neuronal injury in sepsis. Thus, preventing BBB damage is an attractive therapeutic target. Mitochondrial dysfunction is an important contributor of sepsis-induced multi-organ system failure. More recently, mitochondrial dysfunction in endothelial cells has been implicated in mediating BBB failure in stroke, multiple sclerosis and in other neuroinflammatory disorders. Here, we focused on Drp1-mediated mitochondrial dysfunction in endothelial cells as a potential target to prevent BBB failure in sepsis.

Methods: We used lipopolysaccharide (LPS) to induce inflammation and BBB disruption in a cell culture as well as in murine model of sepsis. BBB disruption was assessed by measuring levels of key tight-junction proteins. Brain cytokines levels, oxidative stress markers, and activity of mitochondrial complexes were measured using biochemical assays. Astrocyte and microglial activation were measured using immunoblotting and qPCR. Transwell cultures of brain microvascular endothelial cells co-cultured with astrocytes were used to assess the effect of LPS on expression of tight-junction proteins, mitochondrial function, and permeability to fluorescein isothiocyanate (FITC) dextran. Finally, primary neuronal cultures exposed to LPS were assessed for mitochondrial dysfunction.

Results: LPS induced a strong brain inflammatory response and oxidative stress in mice which was associated with increased Drp1 activation and mitochondrial localization. Particularly, Drp1-(Fission 1) Fis1-mediated oxidative stress also led to an increase in expression of vascular permeability regulators in the septic mice. Similarly, mitochondrial defects mediated via Drp1-Fis1 interaction in primary microvascular endothelial cells were associated with increased BBB permeability and loss of tight-junctions after acute LPS injury. P110, an inhibitor of Drp1-Fis1 interaction, abrogated these defects, thus indicating a critical role for this interaction in mediating sepsis-induced brain dysfunction. Finally, LPS mediated a direct toxic effect on primary cortical neurons, which was abolished by P110 treatment.

Conclusions: LPS-induced impairment of BBB appears to be dependent on Drp1-Fis1-mediated mitochondrial dysfunction. Inhibition of mitochondrial dysfunction with P110 may have potential therapeutic significance in septic encephalopathy.
\end{abstract}

Keywords: Drp1, Neuroinflammation, Blood brain barrier, P110, Sepsis

\footnotetext{
*Correspondence: bhailes3@stanford.edu; aujoshi@stanford.edu Bereketeab Haileselassie, Amit U. Joshi are shared first author.

'Department of Chemical and Systems Biology, Stanford University School of Medicine, Stanford, CA, USA

Full list of author information is available at the end of the article
}

(c) The Author(s). 2020 Open Access This article is distributed under the terms of the Creative Commons Attribution 4.0 International License (http://creativecommons.org/licenses/by/4.0/), which permits unrestricted use, distribution, and reproduction in any medium, provided you give appropriate credit to the original author(s) and the source, provide a link to the Creative Commons license, and indicate if changes were made. The Creative Commons Public Domain Dedication waiver (http://creativecommons.org/publicdomain/zero/1.0/) applies to the data made available in this article, unless otherwise stated. 


\section{Background}

Sepsis, an exaggerated systemic inflammatory response syndrome, has often been associated with multi-organ damage and subsequent increased long-term morbidity and mortality [1, 2]. Sepsis-associated encephalopathy (SAE) is a common but poorly understood neurological complication of sepsis that is often associated with cerebral coagulopathy, ischemia, and inflammation in the brain. Clinically, SAE is linked with a high risk of mortality, and survivors often suffer from chronic autonomic nerve dysfunctions, delirium, or damaged cognitive functions of different degrees [3]. Despite the prevalence and severity of this condition, there is no prophylactic or targeted therapy to abrogate neurologic injury in septic patients, and standard of care is limited to the non-specific supportive measures and treatment of the underlying infection $[4,5]$.

While the pathobiology behind septic encephalopathy is still poorly understood, many mechanisms have been proposed [6, 7]. The current hypothesis suggests that a combination of dysregulated brain perfusion and oxygenation resulting in altered blood-brain-barrier (BBB) contributes significantly to the neurologic dysfunction in sepsis [4, 8-10]. This phenomenon is further enhanced by other contributors, such as renal and/or hepatic dysfunction, disglycemia, fever, and the use of neurotropic drugs, as well as environmental factors [11].

More recently, a focus on "cytopathic hypoxia," a disturbance of cellular bioenergetics in sepsis, has revealed the influence of mitochondrial damage on end-organ failure and eventual mortality [11-14]. Furthermore, the role of mitochondrial failure is associated with dysfunctional $\mathrm{BBB}$ in other neurologic disorders, including stroke, Alzheimer's disease (AD), Parkinson disease (PD), Huntington's disease, and epilepsy [15-19]. Despite these findings, the role of altered mitochondrial dynamics (fission, fusion, and mitophagy), which is key for mitochondrial quality control [20], has not been adequately explored in the context of BBB dysfunction and subsequent septic encephalopathy.

We have previously demonstrated a therapeutic effect of peptide P110, a 7-amino acid peptide representing a homology sequence between dynamin-related protein 1 (Drp1) and Fission 1 (Fis1) [21], in reducing pathological mitochondrial dysfunction in multiple neurodegenerative diseases [20, 22-25]. This project evaluates the role of Drp1/Fis1 interaction in mediating mitochondrial failure and subsequent BBB dysfunction in the setting of sepsis.

\section{Methods}

\section{Animals}

All animal experimental procedures were conducted in accordance with the animal care regulations of the $\mathrm{Na}$ tional Institute of Health and were approved by Stanford
University's Administrative Panel on Laboratory Animal Care. The mice were maintained on a 12-h light/dark cycle in stable conditions in terms of temperature, humidity, and ventilation. Water and food were offered ad libitum. Animals were randomly assigned numbers and evaluated thereafter by a researcher blinded to both experimental condition and genotype.

\section{In vivo model for severe septic injury}

Five to seven-week-old BALB/c mice were randomized into control $(n=4)$, lipopolysaccharide (LPS) $(n=8)$, and LPS + P110-treated $(n=8)$ groups. LPS was administered at $8 \mathrm{mg} / \mathrm{kg}$ to induce a severe sepsis phenotype [26]. Peptide P110 was administered at $0.5 \mathrm{mg} / \mathrm{kg} /$ day (dissolved in $0.2 \mathrm{ml}$ of saline) intraperitoneally, $3 \mathrm{hrs}$ following LPS.

\section{Primary brain microvascular endothelial cells}

Primary brain microvascular endothelial cells (BMECs) were isolated from adult mice according to existing protocols [27]. In brief, BMECs were cultured in Dulbecco's modified Eagle's medium (DMEM)/F12 supplemented with 20\% FBS, $1 \%$ GlutaMAX (Life Technologies), basic fibroblast growth factor (BFGF; $1 \mathrm{ng} / \mathrm{ml}$; Roche Life Sciences), heparin $(100 \mu \mathrm{g} / \mathrm{ml})$, insulin $(5 \mu \mathrm{g} / \mathrm{ml})$, transferrin $(5 \mu \mathrm{g} / \mathrm{ml})$, selenium $(5 \mathrm{ng} / \mathrm{ml})$ (insulin-transferrin-selenium medium supplement; Life Technologies), and gentamicin $(50 \mu \mathrm{g} / \mathrm{ml} ;$ Sigma Aldrich). Puromycin $(4 \mu \mathrm{g} / \mathrm{ml}$; Sigma Aldrich) was added to the BMEC medium for the first $48 \mathrm{~h}$ after plating to remove pericytes and increase endothelial cell purity. Cultures were maintained at $37^{\circ} \mathrm{C}$ in a humidified atmosphere of $5 \% \mathrm{CO} 2 / 95 \%$ air. The purified primary BMECs were used to construct in vitro $\mathrm{BBB}$ model when $80 \%$ confluent.

\section{Primary astrocyte culture}

Primary astrocyte cultures were prepared from cerebral cortices of $\mathrm{P} 0 / \mathrm{P} 1$ pups $(\mathrm{C} 57 \mathrm{BL} / 6)$. In brief, the meninges were carefully removed from cortices using a dissecting microscope. Then, the cleaned cortices were mechanically dissociated in astrocyte culture medium. Astrocytes were grown on poly-L-lysine-coated 24-well plates in high glucose DMEM (Life Technologies) supplemented with $10 \%$ fetal bovine serum [25].

\section{Construction of in vitro BBB model}

BMECs were co-cultured with astrocytes [27]. In brief, BMECs were seeded on the inside of the Transwell insert, which was coated with fibronectin and collagen type IV $\left(0.1 \mathrm{mg} / \mathrm{ml}\right.$, each), at a density of $4 \times 10^{4}$ cells per well. The inserts were then placed in a 24-well plate containing primary astrocytes. The medium used to plate the cells in the 24-well Transwell plate. The medium in the luminal chamber (or the inside of the Transwell insert) was changed $24 \mathrm{~h}$ after seeding. BMEC 
monolayers were cultured for 3 days before use in experiments.

\section{Primary neuron culture}

Primary neuron cultures were prepared from cerebral cortices of embryonic day E17 WT (C57BL/6) [25]. In brief, cortices were dissected and dissociated using papain dissociation system (Worthington Biochemical Corporation). Cells were cultured at 20,000/ well of a 96-well plate coated with poly-D-lysine (Sigma) for cell viability assays. For seahorse experiments, $1 \times 10^{5}$ cells/ well were seeded in XF 24-well cell culture microplate and cultured in Neurobasal medium (Invitrogen) supplemented with B-27 (Invitrogen) containing $25 \mathrm{mM}$ glucose, $4 \mathrm{mM}$ glutamine, $1 \mathrm{mM}$ sodium pyruvate, and $5 \%$ FBS. At $24 \mathrm{~h}$ after seeding, the medium was changed to Neurobasal medium supplemented with B-27 and 0.5 $\mathrm{mM}$ glutamine. Cells were cultured at $37{ }^{\circ} \mathrm{C}$ in a humidified chamber of $95 \%$ air and $5 \% \mathrm{CO}_{2}$. Cultures were used for experiments from 7 to 10 days after seeding.

\section{Trans-endothelial barrier permeability}

Three hours after the treatment with LPS, fluorescein isothiocyanate (FITC)-tagged 150-kDa dextran (Sigma) was added to the upper (luminal) chamber at a final concentration of $0.5 \mathrm{mg} / \mathrm{ml}$ as previously described [28]. Relative fluorescence (485-nm excitation/535-nm emission) was determined at the end of the 24-h incubation period by collection of $100-\mu \mathrm{l}$ aliquots in triplicate from each lower (abluminal) chamber and measurement of fluorescence using SpectraMax M2e (Molecular devices),

\section{Cell and mitochondrial function assays \\ Mitochondrial membrane potential}

Cells were incubated with tetra-methyl-rhodamine methyl ester (200 nM; TMRM, Invitrogen) in HBSS (Hank's balanced salt solution) for $30 \mathrm{~min}$ at $37^{\circ} \mathrm{C}$, as per the manufacturer's protocol, and the fluorescence was analyzed using SpectraMax M2e (Molecular devices, using excitation at $360 \mathrm{~nm}$ and emission at $460 \mathrm{~nm}$ ).

\section{ATP measurements}

Relative intracellular ATP levels were determined using ATP-based Cell Titer-Glo Luminescent Cell Viability kit (Promega), which causes cell lysis and generates a luminescent signal proportional to the amount of ATP present. In brief for intracellular ATP levels, opaquewalled 96-well plates with cell lysate $(50 \mu \mathrm{l})$ were prepared. An equal volume of the single-one-step reagent provided by the kit was added to each well and incubated for $30 \mathrm{~min}$ at room temperature. ATP content was measured using a luminescent plate reader SpectraMax M2e (Molecular devices).

\section{ROS production}

For cellular ROS detection, cells were incubated with 2,7 dichloro-fluorescein diacetate (DCFDA) (Abcam) $100 \mu \mathrm{M}$ for $30 \mathrm{~min}$ at $37^{\circ} \mathrm{C}$ in the dark, and fluorescence was analyzed with excitation/emission at $495 / 529 \mathrm{~nm}$, using SpectraMax M2e (Molecular devices). Fluorescence intensity was then normalized for cell number. To determine mitochondrial ROS production, cells were treated with $5 \mu \mathrm{M}$ MitoSOX ${ }^{\mathrm{TM}}$ Red, a mitochondrial superoxide indicator (Invitrogen) for $10 \mathrm{~min}$ at $37^{\circ} \mathrm{C}$, according to the manufacturer's protocol, and fluorescence was analyzed with excitation/emission at $510 / 580 \mathrm{~nm}$, using SpectraMax M2e (Molecular devices).

\section{Bioenergetic profiles}

Cells were plated in a Seahorse XF24 Cell Culture Microplate (Agilent). All seahorse experiments in neurons were performed at $24 \mathrm{~h}$ after individual stimuli. At the end of the treatment, cells were washed twice with Agilent Seahorse XF Media (Agilent) supplemented with 1 $\mathrm{mM}$ pyruvate, $2 \mathrm{mM} \mathrm{L}$-glutamine, and $2 \mathrm{mM}$ D-glucose; a final volume of $525 \mu \mathrm{l}$ was placed in each well. Cells were then incubated in a $0 \% \mathrm{CO}_{2}$ chamber at $37^{\circ} \mathrm{C}$ for $1 \mathrm{~h}$ before being placed into a Seahorse XFe24 Analyzer (Agilent). For oxygen consumption rate (OCR) and extracellular acidification rate (ECAR) experiments, cells were treated with $1 \mu \mathrm{M}$ oligomycin, $2 \mu \mathrm{M}$ carbonyl cyanide p-trifluoromethoxy phenylhydrazone (FCCP), and $0.5 \mu \mathrm{M}$ rotenone/antimycin. A total of three OCR and $\mathrm{pH}$ measurements were taken after each compound was administered. All seahorse experiments were repeated at least three times.

\section{Cell death}

Cytotoxicity was determined using Cytotoxicity Detection Kit, as before [23]. In brief, media was collected at endpoints (in phenol red-free DMEM) to measure the percentage of released lactate dehydrogenase activity (LDH). To quantify total LDH, cells were lysed with Triton $\mathrm{X}$ (1\% in serum-free cell culture media) overnight at $4{ }^{\circ} \mathrm{C} ; 50 \mu \mathrm{l}$ media or lysate was transferred with $50 \mu \mathrm{l}$ of reaction mix in a 96-well plate and incubated at RT for $30 \mathrm{~min}$ in the dark. Absorbance was measured at $490 \mathrm{~nm}$ using SpectraMax M2e (Molecular devices), and cell death is presented as a percent of released $\mathrm{LDH}$ of total $\mathrm{LDH}$.

\section{Caspase activity assay}

Caspase 3 activities were determined using a colorimetric caspase 3 assay kit (Abcam), according to the manufacturer's protocol. Cell lysates containing $200 \mu \mathrm{g}$ protein were used for each assay. Each assay was repeated with three independent cell cultures. Absorbance at $400 \mathrm{~nm}$ was recorded using SpectraMax M2e (Molecular 
devices). Caspase activity was calculated in arbitrary units and represented as fold change of WT control.

\section{Western blot analysis}

Protein concentrations were determined using the Bradford assay (Thermo Fisher Scientific). Proteins were resuspended in Laemmli buffer containing 2mercaptoethanol, loaded on SDS-PAGE, and transferred on to nitrocellulose membrane, $0.45 \mu \mathrm{m}$ (BioRad), as before [20]. Cell supernatant was cleared of cellular debris by centrifugation at $1000 \mathrm{~g}$ for $10 \mathrm{~min}$. The total lysate was resuspended in Laemmli buffer containing 2-mercaptoethanol, loaded on SDS-PAGE, and transferred on to nitrocellulose membrane, $0.45 \mu \mathrm{m}$ (BioRad), as before [20]. Membranes were cut at appropriate molecular weights and then probed with the indicated antibody and visualized by ECL $(0.225 \mathrm{mM}$ p-coumaric acid; Sigma), 1.25 mM 3-aminophthalhydrazide (Luminol; Fluka) in $1 \mathrm{M}$ Tris $\mathrm{pH}$ 8.5. Scanned images of the exposed $\mathrm{X}$-ray film or images acquired with Azure Biosystems C600 were analyzed with ImageJ to determine relative band intensity. Quantification was performed on samples from independent cultures for each condition.

\section{RNA isolation and gene expression analysis}

RNA isolation was performed using GenElute ${ }^{\mathrm{Tw}}$ Mammalian Total RNA Miniprep Kit (Sigma Aldrich) according to the manufacturer's protocols. RNA concentration was measured using a Nanodrop (ND -1000; NanoDrop Technologies, Rockland, DE, USA), and RNA integrity was assessed using a Bioanalyzer (2100; Agilent Technologies, Palo Alto, CA, USA). cDNA synthesis was performed using the Quantitect reverse transcription kit (Qiagen) according to the manufacturer's instructions, with a minimal input of 200 ng total RNA. Quantitative PCR (qPCR) was performed using the 7300 Real Time PCR system (Applied Biosystems, Foster City, USA) using the equivalent cDNA amount of 1-2 ng total RNA used in cDNA synthesis. SYBRgreen master mix (Applied Biosystems) and a $2 \mathrm{pmol} / \mathrm{ml} \mathrm{mix}$ of forward and reverse primer sequences were used for 40 cycles of target gene amplification.

\section{Statistical analysis}

Prism 8.0 (GraphPad Software) was used for the statistical analysis. Data shown are the mean \pm SD. with $P<0.05$ considered statistically significant. Group differences were analyzed with one-way analysis of variance (ANOVA) followed by Holms-Sidak multiple comparisons test for multiple groups. Data distribution was assumed to be normal, but this was not formally tested. No statistical methods were used to predetermine sample sizes.

\section{Results}

Drp1-Fis1-mediated mitochondrial dysfunction is a key mechanism in LPS-induced brain microvascular permeability

Gene expression profile of vascular integrity and inflammation of primary brain microvascular endothelial cells co-cultured with astrocytes demonstrates a significant shift to a pro-inflammatory phenotype and activation of key mediators of vascular endothelial permeability following LPS treatment $(0.1 \mu \mathrm{g} / \mathrm{ml}$ for $24 \mathrm{hrs})$ (Fig. 1a). This is associated with pathologic mitochondrial Drp1 activation, as measured by phosphorylation at Serine 616 (fold change $4.05 \pm 1.142, p=0.0006$ ), suggesting a shift towards a pro-fission state $[20,23,24,26]$ (Fig. 1b). The mitochondrial damage in microvascular endothelial cells and loss of BBB integrity is correlated with increased mitochondrial specific (MitoSOX; $p=0.002$ ) as well as total $(p=0.002)$ oxidative stress as well as a loss of mitochondrial membrane potential (TMRE; $p<0.001$ ) following LPS treatment (Fig. 1c-f).

LPS-mediated derangement in vascular endothelial function were ameliorated with P110 treatment, represented by limited activation of key enzymes that mediate vascular endothelial permeability as well as limited Drp1 activation and mitochondrial localization. P110 treatment also lead to improvement in BBB integrity and improved mitochondrial health represented by limited oxidative stress (MitoSox; $p=0.02$ ) as well as improved mitochondrial membrane potential (TMRM; $p=0.01$ ) (Fig. 1).

Inhibition of Drp1-Fis1-mediated mitochondrial dysfunction is protective against LPS-mediated neuronal injury in culture

When evaluating the primary neurons, LPS treatment caused a significant mitochondrial damage, leading to a decrease in oxidative phosphorylation (basal OCR; $p=0.0002$ ) and subsequent shift towards glycolysis (basal ECAR; $p=0.0004$ ). Mitochondrial damage was further supported by the decreased mitochondrial membrane potential (TMRE; $p=<0.0001$ ) and ATP production (fold change of control $=0.557 \pm 0.002, p=0.0017$ ) following LPS treatment (Fig. 2a-c). Similar to the brain microvascular endothelial cells, LPS-treated primary neurons had an increase in Drp1 activation and mitochondrial localization (fold change $1.86 \pm 0.31, p=0.003$ ), suggesting a role for Drp1 in the progression of mitochondrial failure in the setting of sepsis. LPS treatment of primary neurons subsequently led to increased p53 association with the mitochondrial membrane (fold change $=1.59 \pm$ $0.26, p=0.0064)$ and increased cell death (LDH (\% of control); $p=0.001$ ).

Further supporting the role of Drp1-Fis1-mediated derangements in mitochondrial dysfunction under LPS stimulation, peptide P110-treated neurons had improved mitochondrial function (basal OCR; $p=0.0112$ ) and 


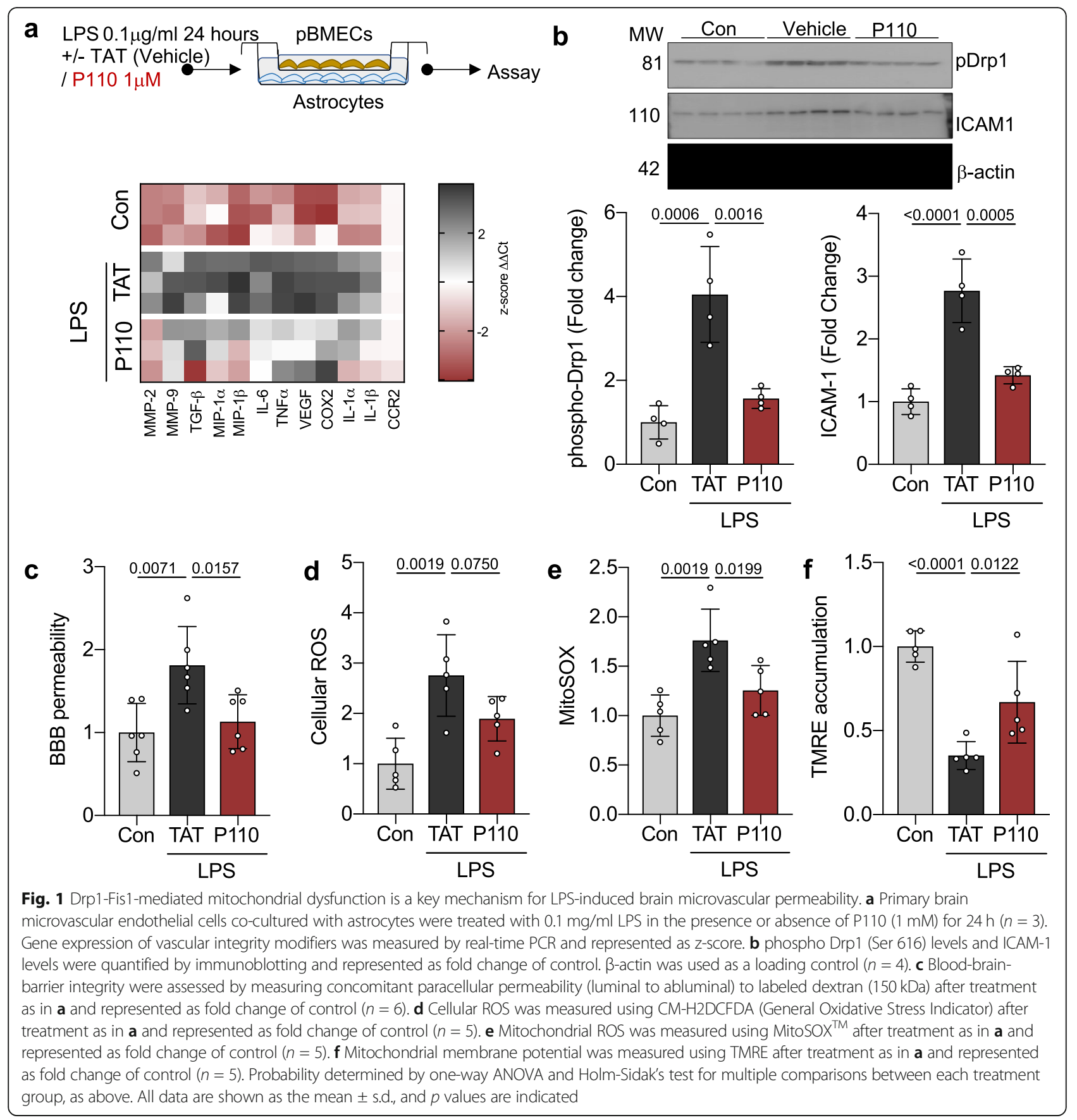

subsequent decreased glycolysis (basal ECAR; $p=0.0365$ ) leading to improved ATP production (fold change of control $=0.857 \pm 0.13, p=0.0199)$. P110 treatment was also noted to abrogate the downstream effects of LPS treatment by limiting p53 activation (mitochondrial p53 fold change $1.23 \pm 0.09, p=0.0462)$ and subsequent cell death (LDH (\% of control); $p=0.0467$ ).

Inhibition of Drp1/Fis1-mediated mitochondrial dysfunction is protective in a mouse model of septic encephalopathy
Using an established LPS model of sepsis (28) in 5 to 7-week-old female BALB/c mice, we evaluated the role of Drp1/Fis1 interaction in sepsis-mediated encephalopathy. Brain tissue from septic mice demonstrated a significant decrease in mitochondrial function, represented by increased oxidative stress (brain $\mathrm{H}_{2} \mathrm{O}_{2} ; p=0.01$ ) as well as decreased ATP levels $(p=0.008)$, which was significantly improved with P110 treatment (Fig. 3b, c). When evaluating $\mathrm{BBB}$ integrity, LPS treatment caused a significant 


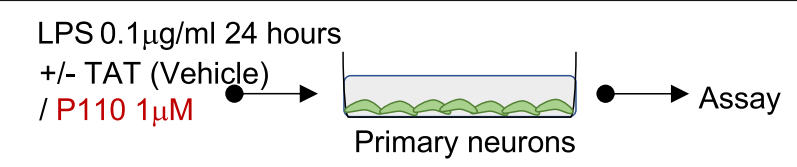

a

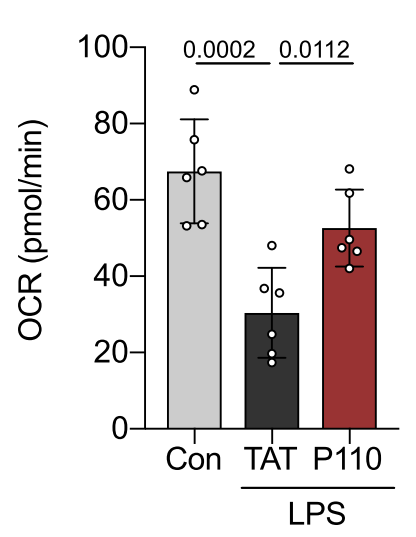

d

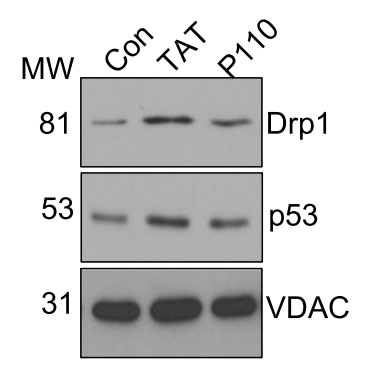

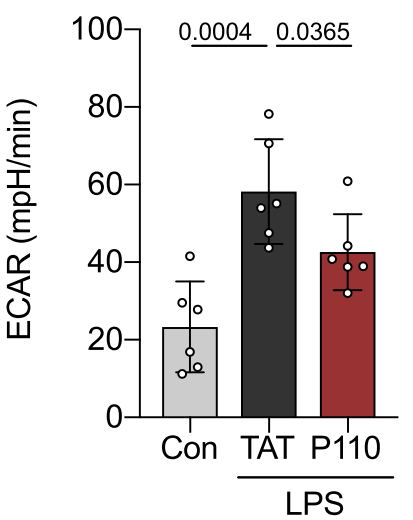

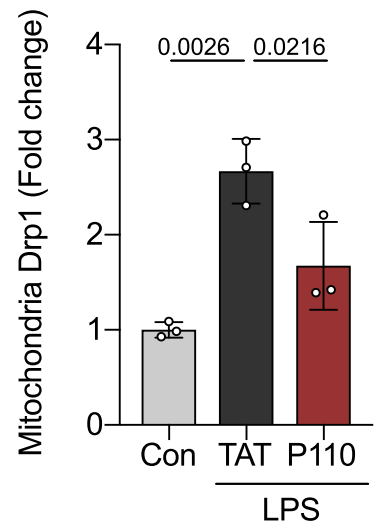

b
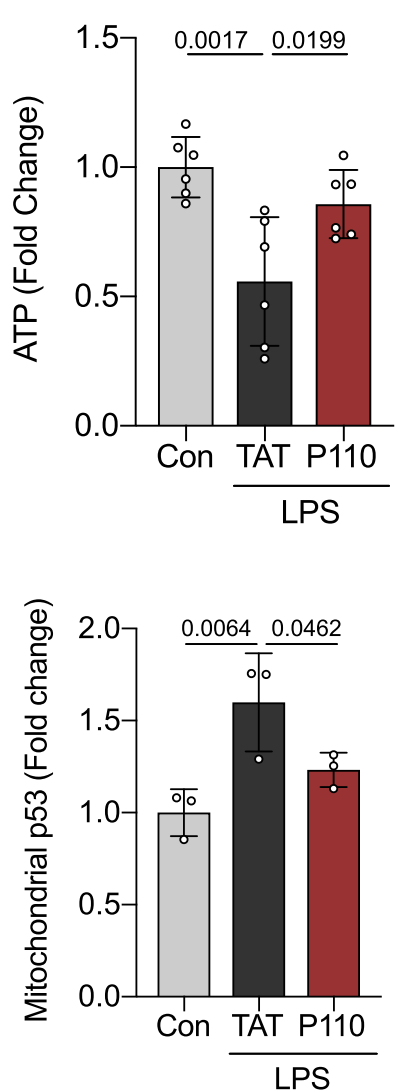

C
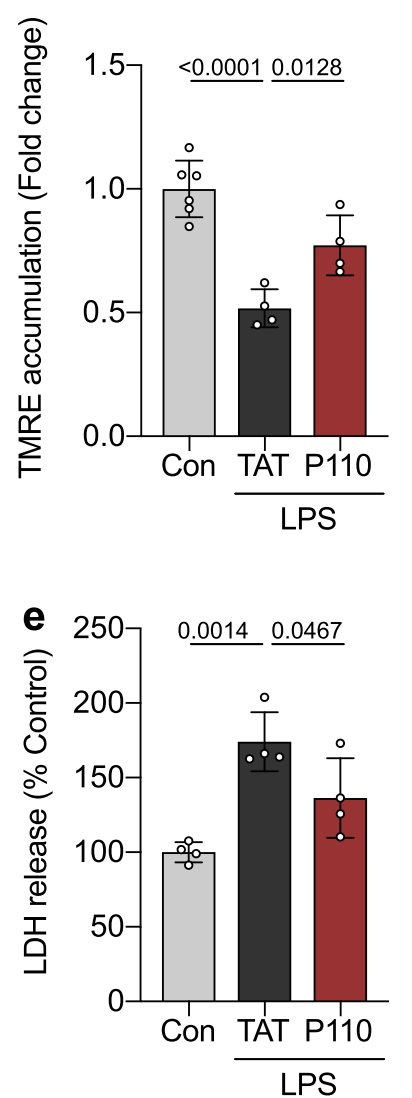

Fig. 2 Inhibition of Drp1-Fis1-mediated mitochondrial dysfunction is protective against LPS-mediated neuronal injury in culture. a Oxidative phosphorylation and glycolytic rate was measured with Seahorse Extracellular Flux in primary neurons were treated with 0.1 mg/ml LPS in the presence/absence of P110 (1 mM) for $24 \mathrm{~h}(n=6)$. b Cellular ATP level was measured using CellTiter-Glo ${ }^{\circledR}$ after treatment as in a and represented as fold change of control. c Mitochondrial membrane potential was measured using TMRE after treatment as in a and represented as fold change of control $(n=6)$. $\mathbf{d}$ Drp1 levels and p53 levels were quantified by immunoblotting in enriched mitochondrial fractions and represented as fold change of control. VDAC was used as a loading control $(n=3)$. e LDH release was measured after treatment as in a and represented as $\%$ of Control (Con) $(n=6)$. Probability determined by one-way ANOVA and Holm-Sidak's test for multiple comparisons between each treatment group, as above. All data are shown as the mean \pm s.d., and $p$ values are indicated

decrease in proteins forming the vascular tight junctions (ZO-1 and occludin) and upregulation of vascular leukocyte adhesion molecules (VCAM-1 and ICAM-1), suggesting a compromise to BBB integrity which was abrogated under P110 treatment (Fig. 3d-h).

Similar to the findings using cell culture model, LPS-mediated derangements in mitochondrial function in brain tissue of septic mice was associated with Drp1 activation, represented by increased Drp1 phosphorylation and mitochondrial localization (fold change $2.801 \pm 0.59 ; p<0.001)$. These increases correlated with activation of cell death pathways, including leak of mitochondrial cytochrome c (fold change $0.52 \pm 0.13, p=0.0015$ ), a potent apoptotic trigger (Fig. $4 \mathrm{a}-\mathrm{c}$ ). Inhibition of Drp1/Fis1 interaction, using P110, reduced the level of released mitochondrial cytochrome $C$ (fold change, LPS $=0.52 \pm 0.13$ vs LPS + p110 $=0.81 \pm 0.12, p=0.33)$ and decreased the level of neuroinflammatory markers, TNF $\alpha$, ILI- $\beta$, and IL-6, seen in septic mice (Fig. 4d-f).

When evaluating morbidity and mortality in this murine model of sepsis, P110 treatment reduced clinical disease severity, evidenced by significantly reduced weight loss and a faster weight recovery (Fig. 4g). Importantly, 
a Animal model for severe sepsis

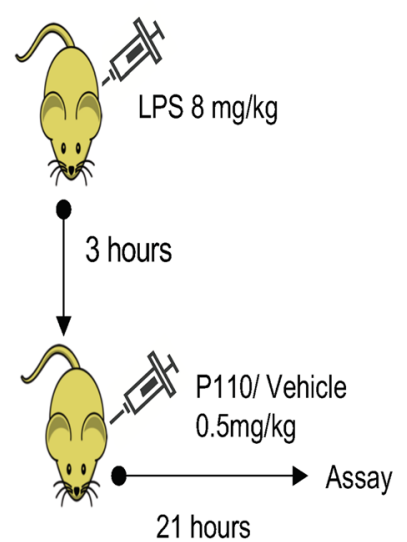

d
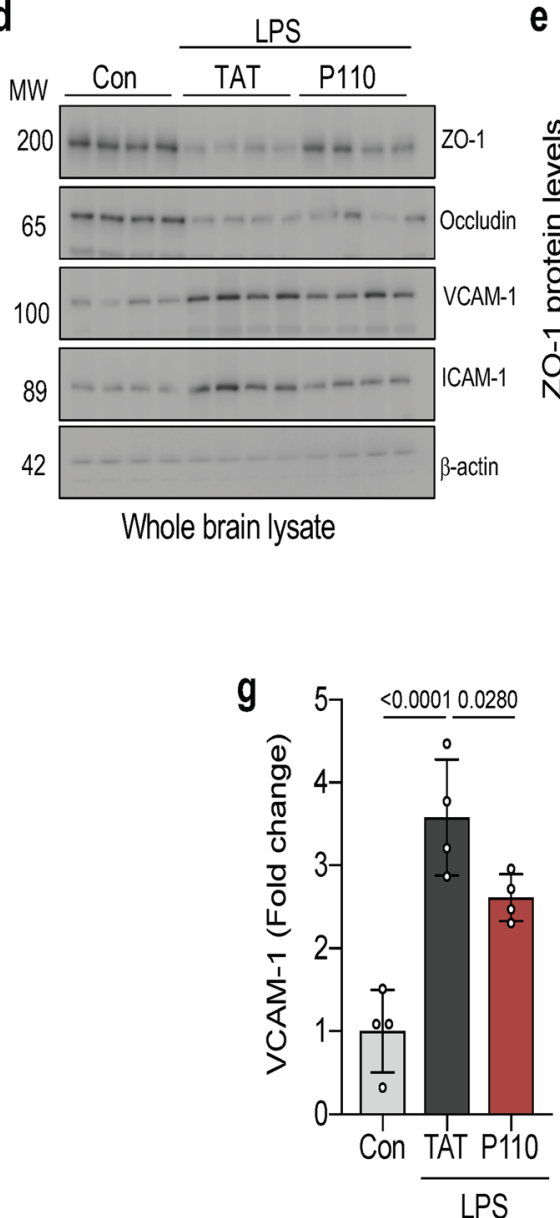

C
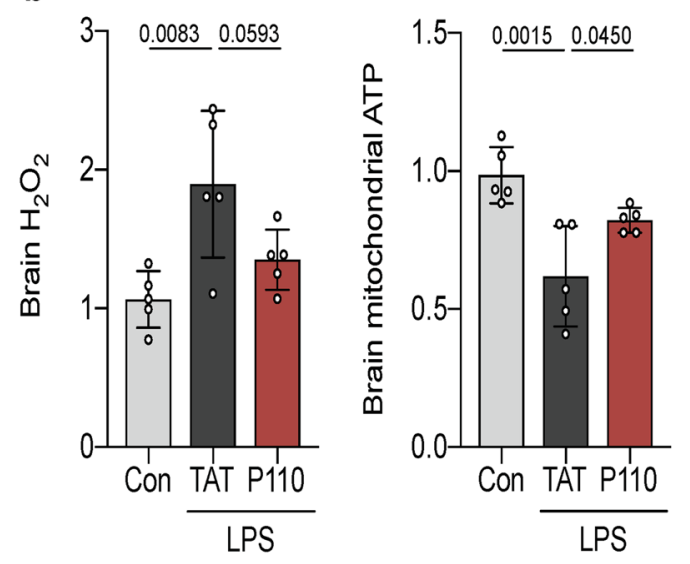

e
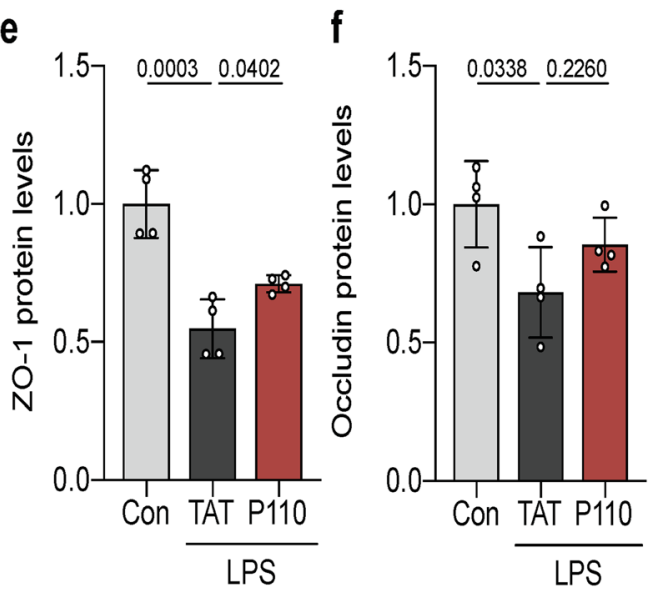

Fig. 3 Inhibition of Drp1-Fis1-mediated mitochondrial dysfunction reduces oxidative stress in the brain and preserves endothelial integrity. a Balb/c mice were treated with LPS $(8 \mathrm{mg} / \mathrm{kg}$ IP) to induce murine model of sepsis. Peptide P110 (0.5 mg/kg IP) was administered in a subset of septic animals $3 \mathrm{~h}$ following LPS treatment and tissue harvested at $24 \mathrm{~h}$. $\mathbf{b}$ Hydrogen peroxide production was determined by Amplex Red ${ }^{\mathrm{TM}}$ from whole brain mitochondria after treatment as in $\mathbf{a}(n=4)$. c ATP colorimetric assay kit was used to measure ATP levels in freshly isolated whole brain mitochondria after treatment as in a $(n=4)$. d ZO-1, occludin, VCAM-1, and ICAM-1 levels were quantified by immunoblotting. $\beta$ actin was used as a loading control $(n=4)$. e ZO-1, f occludin, $\mathbf{g}$ VCAM-1, and $\mathbf{h}$ ICAM-1 protein levels were quantified and represented as fold change $(n=4)$. Probability determined by one-way ANOVA and Holm-Sidak's test for multiple comparisons between each treatment group, as above. All data are shown as the mean \pm s.d., and $p$ values are indicated 

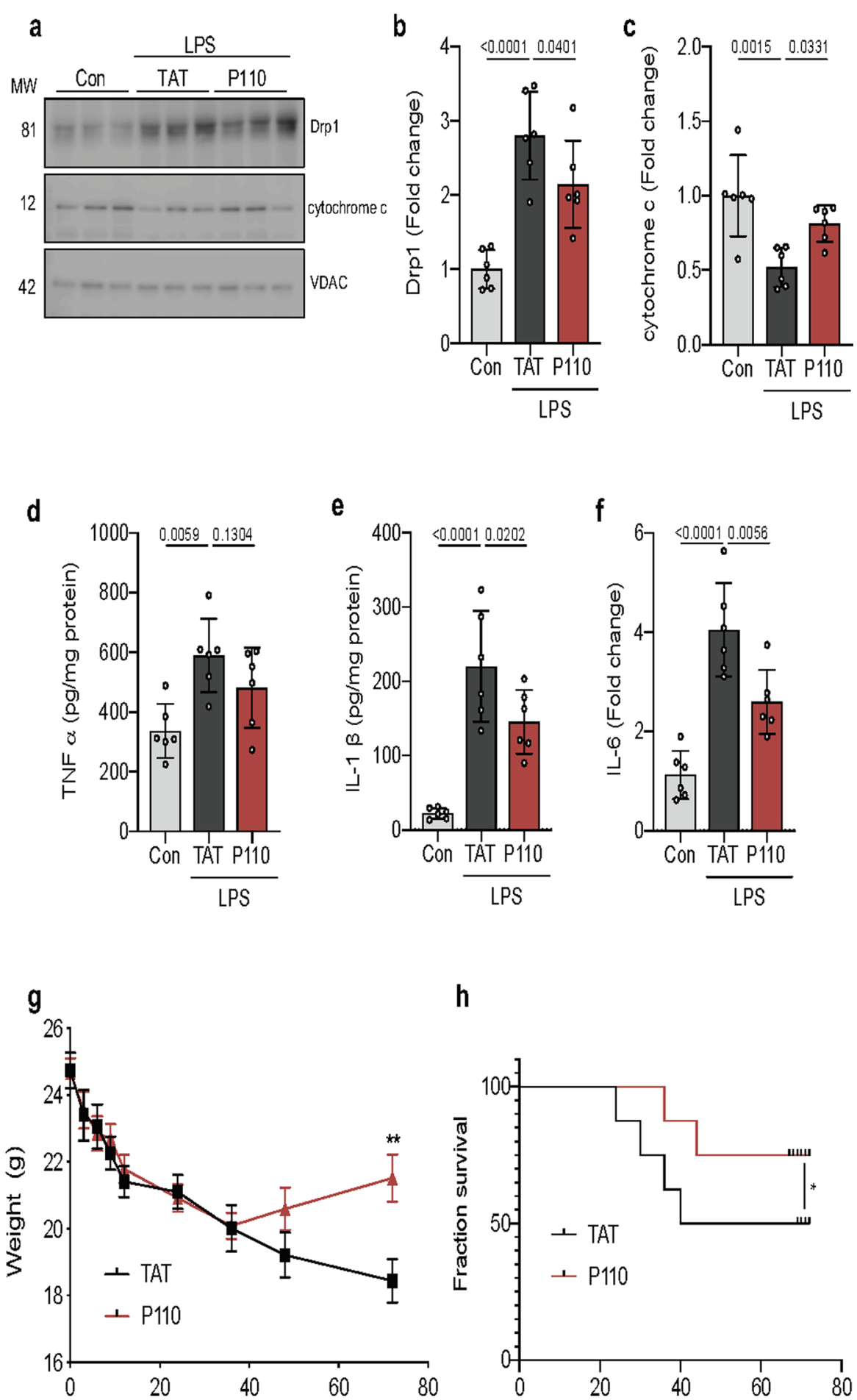

$\mathbf{h}$

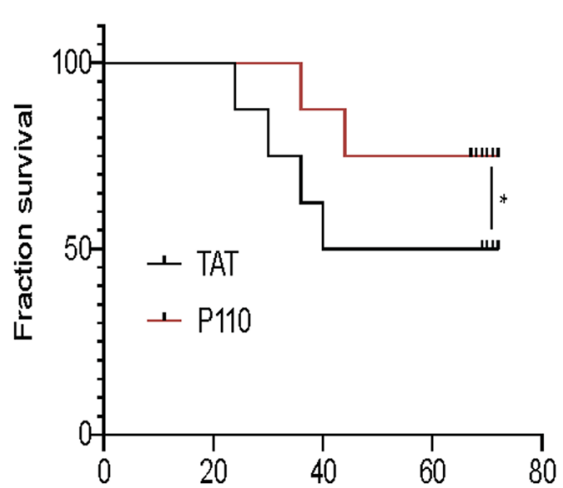

Fig. 4 Inhibition of Drp1-Fis1-mediated mitochondrial dysfunction is protective in mouse model of septic encephalopathy. a Mitochondrial Drp1 translocation and cytochrome $\mathrm{c}$ release were quantified by immunoblotting in enriched mitochondrial fractions. VDAC was used as a loading control. b Drp1 and c cytochrome c protein levels were quantified and represented as fold change $(n=6)$. d TNFa, e IL-1 $\beta$, and $\mathbf{f} \mid L-6$ cytokine levels were measured in whole brain lysate by ELISA after a 24-h treatment of LPS $(n=6)$. $\mathbf{g}$ Weight changes in these mice were quantified as a surrogate for illness severity. ( $n=5$ mice for control, 5 for LPS-treated mice, and 5 for LPS + P1 10-treated). $\mathbf{h}$ Kaplan-Meier survival curve of mice showing increased survival following P110 treatment (red trace) as compared to the trans-activator of transcription (TAT)-treated control mice (black trace). Probability determined by one-way ANOVA and Holm-Sidak's test for multiple comparisons between each treatment group, as above. All data are shown as the mean \pm s.d., and $p$ values are indicated 
P110 treatment reduced mortality by $50 \%$ at $48 \mathrm{~h}$ (which was sustained through $94 \mathrm{~h}$ ) relative to LPS treatment alone (Fig. 4h).

\section{Discussion}

Sepsis-associated encephalopathy (SAE) is a prevalent complication of sepsis. Currently, it is thought to affect over $75 \%$ patients with sepsis $[7,12]$ and causes a higher risk of mortality in those who are afflicted. Additionally, the long-term sequelae for those who survive (which include cognitive impairment, hippocampal atrophy, white matter disease, and ischemic strokes) confer significant limitations on quality of life and incurs a staggering healthcare cost $[1,3,9,29]$.

The pathophysiology of SAE is complex and involves brain microvascular cell dysfunction, loss of blood-brain barrier integrity, microglia and astrocytic activation, and neuronal death [3]. However, not much is known about the role of mitochondrial health in the pathogenesis of sepsis. In the present study, we demonstrate that Drp1 activation and subsequent mitochondrial dysfunction plays a critical role in propagating LPS-mediated SAE using cell culture models of blood brain barrier as well as a mouse model of severe sepsis. In particular, we demonstrate that selective inhibition of Drp1/Fis1 interaction, using P110, reduces $\mathrm{BBB}$ dysfunction and improves neuronal health in culture models. Furthermore, treatment with P110 $3 \mathrm{~h}$ after LPS injection improved mitochondrial health and reduced inflammation in the brain, and improved survival in a mouse model of severe sepsis.

The role of mitochondrial health as a mechanistic link between inflammation and cellular damage in neuroinflammatory and neurodegenerative diseases has become increasingly recognized. In stroke models, LPS-induced derangement in mitochondrial health exacerbates infarct volume and opens BBB in mice subjected to a transient occlusion of the mid carotid artery [15]. Furthermore, LPS increases the paracellular permeability of brain endothelial cells and decreases trans-endothelial electrical resistance [30]. Brain endothelial cells lining the cerebral micro-vessels constitute a physical barrier between blood and brain tissue by a complex network of tight junctions consisting occludin, claudins, junctional adhesion molecules (JAMs), and zonula occludens (ZO)1,2 , and 3 [31]. Of these proteins, $\mathrm{ZO}-1$ is critical in maintaining the junction assembly and its absence has been shown to correlate with BBB failure [32]. Additionally, alteration of the vascular endothelial permeability is thought to result from the release of cytokines, free radicals, matrix metalloproteinases, and nitric oxide [33].

In the in vitro BBB model, we observed that LPS treatment robustly upregulated the gene expression of several pro-inflammatory markers, IL-6, TNF $\alpha$, IL-1 $\alpha$, and IL1- $\beta$ as well as vascular modifiers MMP-2, MMP-9, and
TGF- $\beta$ [33]. These changes are associated with alterations in mitochondrial dynamics as evidenced by temporal association with Drp1 phosphorylation (activation) and mitochondrial fragmentation. Furthermore, the reversal of LPS-mediated changes in proteins that modify vascular permeability and subsequent preservation of $\mathrm{BBB}$ integrity in the presence of P110 further supports the causal association between mitochondrial health and brain microvascular endothelial cell function.

Our earlier studies indicated a protective effect of P110 in multiple neuronal cell models [20, 21, 23]. Similarly, in the present study, we demonstrate a beneficial effect of blocking Drp1/Fis1 interaction on primary neurons exposed to LPS. Mitochondria are critical to many neuronal functions, and any alterations in their health have deleterious effects [34]. LPS interferes with mitochondrial transcription and oxidative phosphorylation (OXPHOS) [35]. Similarly, we observed a decrease in cellular respiration and a shift towards glycolysis under LPS stimulation. However, this metabolic shift did not compensate for the ATP production, subsequently leading to further loss of mitochondrial membrane potential, propagation of Drp1 and p53 recruitment to the mitochondrial outer membrane, and initiation of cell death pathways. All these effects are greatly blunted by P110 treatment as demonstrated by increased ATP levels as well as by improved cell survival.

Similar to the cell culture model used here, injection of a high dose of LPS resulted in an overall decrease in mitochondrial ATP as well as increased ROS levels in the mouse brain. Additionally, the observed Drp1/Fis1dependent effect on intercellular adhesion molecule-1 (ICAM-1) and vascular cell adhesion molecule-1 (VCAM1) suggests a connection between mitochondrial function and inflammatory cell adherence/transmigration across the BBB [36]. Impotently, LPS-mediated changes in brain mitochondrial function were greatly reduced by $\mathrm{P} 110$ treatment, as demonstrated by abrogation in brain vascular endothelial dysfunction, improvement in mouse illness severity (represented by weight loss), and survival.

\section{Conclusion}

In conclusion, we demonstrate Drp1/Fis1 interaction as the mechanism responsible for SAE. Our study identified a potentially new therapeutic strategy to reduce the substantial risk of SAE-associated mortality and morbidity. Future large animal and clinical studies are necessary to evaluate the significance of this mechanistic pathway and therapeutic target in abrogating neuronal injury in the setting of sepsis.

\section{Abbreviations}

AD: Alzheimer's disease; ANOVA: Analysis of variance; BBB: Blood-brainbarrier; BFGF: Basic fibroblast growth factor; BMEC: Brain microvascular endothelial cells; DCFDA: Dichloro-fluorescein diacetate; DMEM: Dulbecco's 
modified Eagle's medium; Drp1: Dynamin-related protein 1; ECAR: Extracellular acidification rate; Fis1: Fission 1; FITC: Fluorescein isothiocyanate; ICAM-1: Intercellular adhesion molecule-1; JAMs: Junctional adhesion molecules; LDH: Lactate dehydrogenase activity; LPS: Lipopolysaccharide; OCR: Oxygen consumption rate; PD: Parkinson disease; qPCR: Quantitative PCR; SAE: Sepsis-associated encephalopathy; TAT: Trans-activator of transcription; TMRM: Tetra-methyl-rhodamine methy ester; VCAM-1: Vascular cell adhesion molecule-1; ZO: Zonula occludens

\section{Acknowledgements}

Not applicable

\section{Authors' contributions}

$\mathrm{AUJ}, \mathrm{BH}$, and DM-R generated the hypothesis and experimental design. KIA contributed to the experimental design. AUJ, BH, RM, and PSM conducted the experiments and helped with the data analysis. All the authors reviewed and edited the final manuscript. All authors read and approved the final manuscript.

\section{Funding}

This work was supported, in part, by NIH HL52141 to D.M-R; Stanford Maternal Child Health Research Institute Pilot Early Career award and 5K12HD047349 to BH; by Paul \& Daisy Soros Fellowship to PSM; and by AG058047 to KIA.

\section{Availability of data and materials}

The datasets used and/or analyzed during the current study are available from the corresponding author on reasonable request.

\section{Ethics approval}

All animal experimental procedures were conducted in accordance with the animal care regulations of the National Institute of Health and were approved by Stanford University's Administrative Panel on Laboratory Animal Care.

\section{Consent for publication}

Not applicable

\section{Competing interests}

$\mathrm{AUJ}$ and DM-R hold patents related to P110 in neurodegenerative diseases. The other authors declare that they do not have competing interests.

\section{Author details}

'Department of Chemical and Systems Biology, Stanford University School of Medicine, Stanford, CA, USA. ${ }^{2}$ Department of Pediatrics, Stanford University School of Medicine, Stanford, CA, USA. ${ }^{3}$ Department of Neurology \& Neurological Sciences, Stanford School of Medicine, Stanford, CA, USA.

\section{Received: 10 October 2019 Accepted: 23 December 2019}

\section{Published online: 27 January 2020}

\section{References}

1. Rossaint J, Zarbock A. Pathogenesis of multiple organ failure in sepsis. Crit Rev Immunol. 2015;35:277-91.

2. Livingston DH, Mosenthal AC, Deitch EA. Sepsis and multiple organ dysfunction syndrome: a clinical-mechanistic overview. New Horiz. 1995;3:257-66.

3. Widmann CN, Heneka MT. Long-term cerebral consequences of sepsis. Lancet Neurol. 2014;13:630-6.

4. Nwafor DC, Brichacek AL, Mohammad AS, Griffith J, Lucke-Wold BP, Benkovic SA, Geldenhuys WJ, Lockman PR, Brown CM. Targeting the bloodbrain barrier to prevent sepsis-associated cognitive impairment. J Cent Nerv Syst Dis. 2019;11:1179573519840652.

5. Gustot T. Multiple organ failure in sepsis: prognosis and role of systemic inflammatory response. Curr Opin Crit Care. 2011:17:153-9.

6. Ziaja M. Septic encephalopathy. Curr Neurol Neurosci Rep. 2013;13:383.

7. Gofton TE, Young GB. Sepsis-associated encephalopathy. Nat Rev Neurol. 2012:8:557-66.

8. Jeppsson B, Freund HR, Gimmon Z, James JH, von Meyenfeldt MF, Fischer JE. Blood-brain barrier derangement in sepsis: cause of septic encephalopathy? Am J Surg. 1981;141:136-42.

9. Kuperberg SJ, Wadgaonkar R. Sepsis-Associated Encephalopathy: The bloodbrain barrier and the sphingolipid rheostat. Front Immunol. 2017;8:597.
10. Nishioku T, Dohgu S, Takata F, Eto T, Ishikawa N, Kodama KB, Nakagawa S, Yamauchi A, Kataoka Y. Detachment of brain pericytes from the basal lamina is involved in disruption of the blood-brain barrier caused by lipopolysaccharideinduced sepsis in mice. Cell Mol Neurobiol. 2009:29:309-16.

11. Singer M. The role of mitochondrial dysfunction in sepsis-induced multiorgan failure. Virulence. 2014;5:66-72.

12. Duran-Bedolla J, Montes de Oca-Sandoval MA, Saldana-Navor V, VillalobosSilva JA, Rodriguez MC, Rivas-Arancibia S: Sepsis, mitochondrial failure and multiple organ dysfunction. Clin Invest Med 2014, 37:E58-E69.

13. Zapelini PH, Rezin GT, Cardoso MR, Ritter C, Klamt F, Moreira JC, Streck EL, Dal-Pizzol F. Antioxidant treatment reverses mitochondrial dysfunction in a sepsis animal model. Mitochondrion. 2008;8:211-8.

14. d'Avila JC, Santiago AP, Amancio RT, Galina A, Oliveira MF, Bozza FA. Sepsis induces brain mitochondrial dysfunction. Crit Care Med. 2008;36:1925-32.

15. Doll DN, Hu H, Sun J, Lewis SE, Simpkins JW, Ren X. Mitochondrial crisis in cerebrovascular endothelial cells opens the blood-brain barrier. Stroke. 2015:46:1681-9.

16. Sweeney MD, Sagare AP, Zlokovic BV. Blood-brain barrier breakdown in Alzheimer disease and other neurodegenerative disorders. Nat Rev Neurol. 2018;14:133-50

17. Drouin-Ouellet J, Sawiak SJ, Cisbani G, Lagace M, Kuan WL, Saint-Pierre M, Dury RJ, Alata W, St-Amour I, Mason SL, et al. Cerebrovascular and bloodbrain barrier impairments in Huntington's disease: potential implications for its pathophysiology. Ann Neurol. 2015;78:160-77.

18. Gray MT, Woulfe JM. Striatal blood-brain barrier permeability in Parkinson's disease. J Cereb Blood Flow Metab. 2015;35:747-50.

19. Kirk J, Plumb J, Mirakhur M, McQuaid S. Tight junctional abnormality in multiple sclerosis white matter affects all calibres of vessel and is associated with blood-brain barrier leakage and active demyelination. J Pathol. 2003;201:319-27.

20. Joshi AU, Saw NL, Shamloo M, Mochly-Rosen D. Drp1/Fis1 interaction mediates mitochondrial dysfunction, bioenergetic failure and cognitive decline in Alzheimer's disease. Oncotarget. 2018;9:6128-43.

21. Qi X, Qvit N, Su YC, Mochly-Rosen D. A novel Drp1 inhibitor diminishes aberrant mitochondrial fission and neurotoxicity. J Cell Sci. 2013;126:789-802.

22. Disatnik MH, Joshi AU, Saw NL, Shamloo M, Leavitt BR, Qi X, Mochly-Rosen D. Potential biomarkers to follow the progression and treatment response of Huntington's disease. J Exp Med. 2016;213:2655-69.

23. Joshi AU, Saw NL, Vogel H, Cunnigham AD, Shamloo M, Mochly-Rosen D. Inhibition of Drp1/Fis1 interaction slows progression of amyotrophic lateral sclerosis. EMBO Mol Med. 2018;10.

24. Joshi AU, Ebert AE, Haileselassie B, Mochly-Rosen D. Drp1/Fis1-mediated mitochondrial fragmentation leads to lysosomal dysfunction in cardiac models of Huntington's disease. J Mol Cell Cardiol. 2019;127:125-33.

25. Joshi AU, Minhas PS, Liddelow SA, Haileselassie B, Andreasson KI, Dorn GW 2nd, Mochly-Rosen D. Fragmented mitochondria released from microglia trigger A1 astrocytic response and propagate inflammatory neurodegeneration. Nat Neurosci. 2019;22:1635-48.

26. Haileselassie B, Mukherjee R, Joshi AU, Napier BA, Massis LM, Ostberg NP, Queliconi BB, Monack D, Bernstein D, Mochly-Rosen D. Drp1/Fis1 interaction mediates mitochondrial dysfunction in septic cardiomyopathy. J Mol Cell Cardiol. 2019;130:160-9.

27. Enzmann G, Mysiorek C, Gorina R, Cheng YJ, Ghavampour S, Hannocks MJ, Prinz V, Dirnagl U, Endres M, Prinz M, et al. The neurovascular unit as a selective barrier to polymorphonuclear granulocyte (PMN) infiltration into the brain after ischemic injury. Acta Neuropathol. 2013;125:395-412.

28. Van Skike $C E$, Jahrling JB, Olson AB, Sayre NL, Hussong SA, Ungvari Z, Lechleiter JD, Galvan V. Inhibition of mTOR protects the blood-brain barrier in models of Alzheimer's disease and vascular cognitive impairment. Am J Physiol Heart Circ Physiol. 2018;314:H693-703.

29. Crippa IA, Subira C, Vincent JL, Fernandez RF, Hernandez SC, Cavicchi FZ, Creteur J, Taccone FS. Impaired cerebral autoregulation is associated with brain dysfunction in patients with sepsis. Crit Care. 2018;22:327.

30. Banks WA, Gray AM, Erickson MA, Salameh TS, Damodarasamy M, Sheibani N, Meabon JS, Wing EE, Morofuji Y, Cook DG, Reed MJ. Lipopolysaccharideinduced blood-brain barrier disruption: roles of cyclooxygenase, oxidative stress, neuroinflammation, and elements of the neurovascular unit. J Neuroinflammation. 2015;12:223.

31. Luissint AC, Artus C, Glacial F, Ganeshamoorthy K, Couraud PO. Tight junctions at the blood brain barrier: physiological architecture and diseaseassociated dysregulation. Fluids Barriers CNS. 2012;9:23. 
32. Stamatovic SM, Johnson AM, Keep RF, Andjelkovic AV. Junctional proteins of the blood-brain barrier: new insights into function and dysfunction Tissue Barriers. 2016;4:e1154641.

33. Spraque AH, Khalil RA. Inflammatory cytokines in vascular dysfunction and vascular disease. Biochem Pharmacol. 2009;78:539-52.

34. Nunnari J, Suomalainen A. Mitochondria: in sickness and in health. Cell. 2012;148:1145-59.

35. Suliman HB, Carraway MS, Welty-Wolf KE, Whorton AR, Piantadosi CA. Lipopolysaccharide stimulates mitochondrial biogenesis via activation of nuclear respiratory factor-1. J Biol Chem. 2003;278:41510-8.

36. Gorina R, Lyck R, Vestweber D, Engelhardt B. beta2 integrin-mediated crawling on endothelial ICAM-1 and ICAM-2 is a prerequisite for transcellular neutrophil diapedesis across the inflamed blood-brain barrier. J Immunol. 2014;192:324-37.

\section{Publisher's Note}

Springer Nature remains neutral with regard to jurisdictional claims in published maps and institutional affiliations.

Ready to submit your research? Choose BMC and benefit from:

- fast, convenient online submission

- thorough peer review by experienced researchers in your field

- rapid publication on acceptance

- support for research data, including large and complex data types

- gold Open Access which fosters wider collaboration and increased citations

- maximum visibility for your research: over $100 \mathrm{M}$ website views per year

At $\mathrm{BMC}$, research is always in progress.

Learn more biomedcentral.com/submissions 